\title{
WCDMA Uplink Parallel Interference Cancellation-System Simulations and Prototype Field Trials
}

\author{
Bo Hagerman \\ Ericsson Research, Ericsson AB, 16480 Stockholm, Sweden \\ Email:bo.hagerman@ericsson.com

\section{Fredrik Gunnarsson} \\ Ericsson Research, Ericsson AB, 58117 Linköping, Sweden
}

Email: fredrik.gunnarsson@ericsson.com

\section{Hideshi Murai}

Nippon Ericsson K.K., Tokyo 112-0004, Japan

Email: hideshi.murai@ericsson.com

\section{Mioko Tadenuma}

Nippon Ericsson K.K., Tokyo 112-0004, Japan

Email:mioko.tadenuma@ericsson.com

\author{
Jonas Karlsson \\ Ericsson Research, Ericsson AB, 16480 Stockholm, Sweden \\ Email: jonas.b.karlsson@ericsson.com
}

Received 1 March 2004; Revised 20 September 2004

\begin{abstract}
Interference cancellation (IC) is one identified key technology to enhance WCDMA uplink performance. The goal of this contribution is to highlight the relative uplink system capacity improvement available for WCDMA, especially in realistic typical urban radio environments when employing receiver implementations including realistic channel estimation, searcher, and so forth. The performance of the selected limited-complexity parallel IC receiver is first evaluated with link-level simulations in order to provide input to system-level simulations. The system-level methodology is explained and a $40 \%$ system-level uplink capacity increase compared to utilizing the conventional RAKE receiver is found. The limited-complexity parallel IC receiver is then evaluated in a single-cell field trial. The trials show that both the mean and the variance of the outer-loop power control is reduced, which implies an overall increased capacity and an increased battery life of the terminals. Furthermore, the observed capacity gains are in accordance with system simulations.
\end{abstract}

Keywords and phrases: CDMA, field trials, interference cancellation, link-level simulations, system-level simulations.

\section{INTRODUCTION}

Interference canceling (IC) is regarded as one of the key technologies for enhancing CDMA uplink performance. The general interest in IC started after Verdú published his

This is an open access article distributed under the Creative Commons Attribution License, which permits unrestricted use, distribution, and reproduction in any medium, provided the original work is properly cited. results on the optimal receiver for Gaussian multiple-access channels [1].

Several studies have been published on suboptimal and less complex receivers, for example, a class of linear receivers [2], successive cancellation schemes [3], and particularly on parallel IC (PIC) schemes $[4,5,6,7,8,9]$. However, these studies are mainly focused on link performance or using an ideal analytical system approach. Realistic channel models [10] and dynamic behavior in a system environment have 


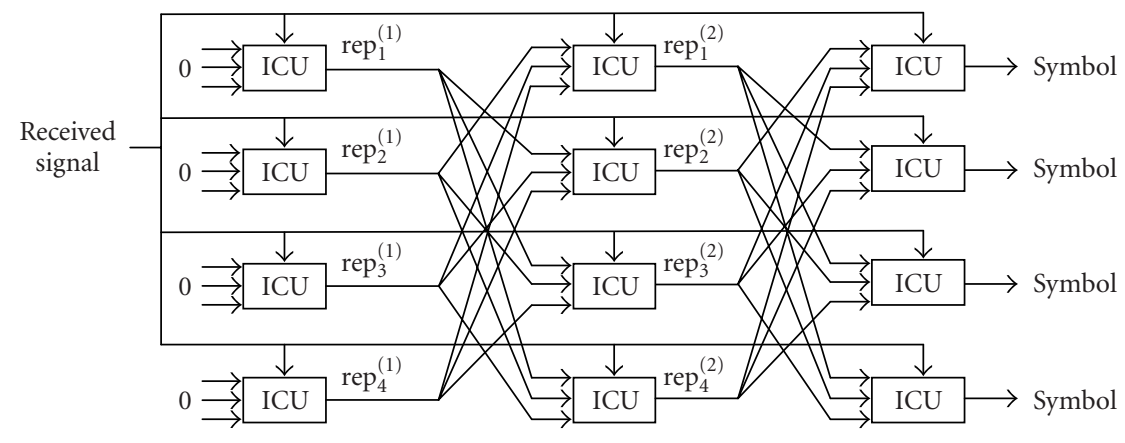

Figure 1: PIC layout.

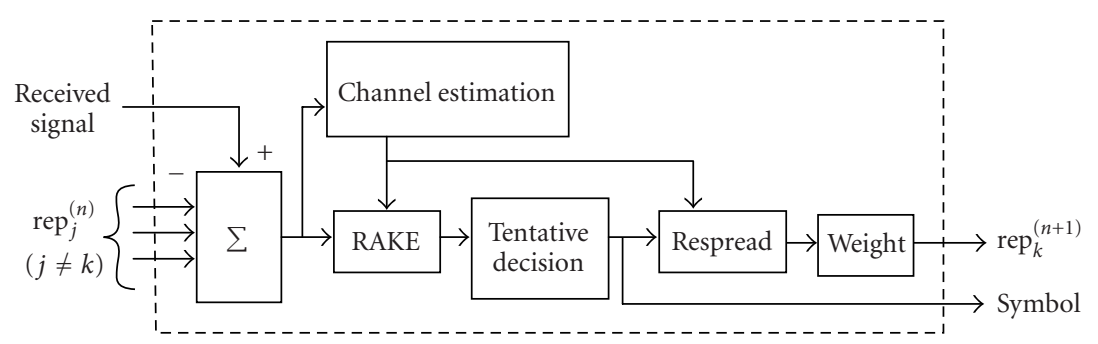

FIgURe 2: ICU layout.

been less thoroughly covered in the literature. These types of components are important to model, analyze, and understand how to utilize IC in systems to ensure the UL/DL capacity balance and improve the robustness of realistic system implementations.

This paper investigates system-level performance of interference cancellation for the WCDMA uplink [11] by means of simulations and measurements from a prototype PIC implementation. The prototype PIC test system development and the field trial were performed in collaboration between Ericsson, China Academy of Telecommunications Technology (CATT), and Datang Telecom Technology Co. Ltd. The field measurements were performed at the northwest part of urban Beijing, China.

The link-level simulator employs a realistic COST 259 channel model [10] and a complete WCDMA receiver including searcher, channel estimation, coding, and so forth in addition to IC functionality. The system-level simulator models user dynamics such as power control, mobility, soft handover, and so forth. The test system utilizes a prototype PIC multiuser receiver implementation integrated into a commercial Ericsson RBS 3202 WCDMA radio base station. The PIC simulator model has been carefully designed to be equivalent to the prototype implementation.

This paper has been organized as follows. First an algorithm description segment with PIC algorithm details and a description of IC interaction with a multicell system are presented. Then, the simulation workflow is described as an iterative procedure of link and system simulations, together with simulated capacity results. Finally, test system details and field measurements prerequisites are presented, and the trial system performance is evaluated, followed by some conclusive remarks.

\section{PARALLEL IC ALGORITHM}

A PIC consists mainly of several cascaded detection units (e.g., RAKE receivers) for each user, see Figure 1, where each detection unit after detection regenerates a replica of the signal based on the detected symbols, estimated channel responses, and the user's spreading codes. These detection units, denoted by interference cancellation units (ICUs) shown in Figure 2, receive as input all the other active users' signal replicas from the previous stage in the cascaded chain of ICUs. As is visible in Figure 1, the original total received signal is also one input to the ICU.

Within the ICU, the replicas are subtracted from the original total received signal and a tentative symbol decision is formed using a standard RAKE receiver and channel response estimator. The channel response estimator averages the pilot symbols from two consecutive slots of the WCDMA uplink signal in order to form an estimate. The ICU output consists of the tentative symbol decision together with a weighted form of the replica signal.

Three cascaded ICU stages have been selected for the PIC algorithm investigated in this paper as well as for the prototype implementation. The weighting factor utilized for each stage should reflect the confidence in the tentative decision $[12,13]$. No replica is generated in the third stage, and therefore there is no weighting factor. A 2D search, using link-level 


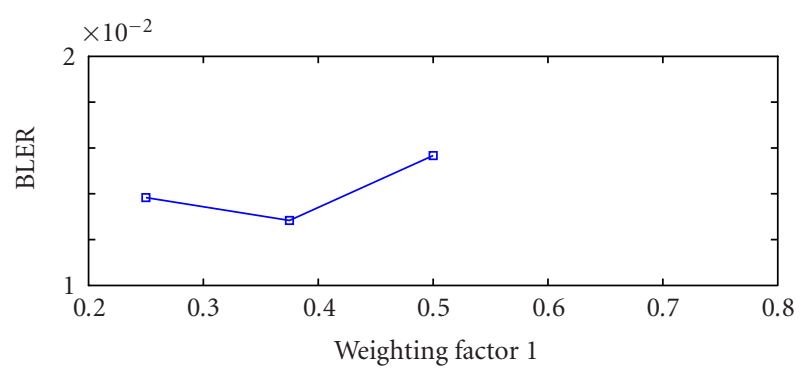

(a)

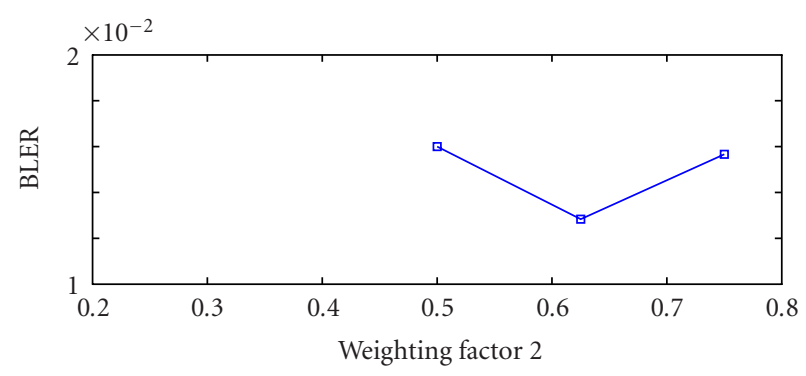

(b)

FIgURE 3: Two slices of the 2D search for optimal weighting factors are presented. (a) shows the block error rate (BLER) as function of weighting factor 1 when weighting factor 2 is set to 0.625 . (b) shows the BLER as a function of weighting factor 2 when weighting factor 1 is set to 0.375 .

simulations, was done in order to find the optimal weighting factors. The optimal weighting factors for the first and second stages were found to be 0.375 and 0.625 , respectively; see Figure 3.

\section{PIC INTERACTION WITH SYSTEM PERFORMANCE}

All users will, dependent on their service, have a quality requirement on the dedicated communication link. In WCDMA, the control mechanism to ensure the fulfillment of the quality requirement is mainly power control $(P C)$. The WCDMA PC is performed on two levels, the inner-loop and outer-loop PC. The inner-loop PC operates at $1500 \mathrm{~Hz}$ to follow fast channel variations. The outer-loop PC evaluates the service quality on higher layer (i.e., on a longer time scale) and sets the target for the inner-loop PC accordingly.

In a multiple-cell deployment, interference is generated both from users in surrounding cells (intercell interference) as well as from users in the own cell (intracell interference). The PIC algorithm is of course effective only towards intracell interference. Thus, the PIC will reduce the impact of the intracell interference compared to a conventional RAKE receiver and thereby via the PC reduce the required output power of the UEs to maintain the service quality.

The typical uplink radio interface load measure is the noise rise, that is, the ratio between total received wideband power at the base station $P$ and the noise power $N$. A popular model is to introduce the fractional load $L[14]$ as

$$
\frac{P}{N}=\frac{1}{1-L}=\frac{1}{1-M / M_{p}}
$$

where $M$ is the number of users and $M_{p}$ is the pole capacity-essentially the upper limit of the users the network can accommodate. In the single-cell case, it is straightforward to derive an expression for $L$. The received wideband power can be expressed as

$$
P=\sum_{m=1}^{M} p_{m} g_{m}+N
$$

where $m$ denotes the $m$ th user equipment (UE) in the cell, $p_{m}$ is the UE power, and $g_{m}$ the power gain from UE to the base station. Furthermore, the carrier-to-interference ratio, CIR (defined here as dedicated physical control channel (DPCCH) received signal code power relative to the received wideband power), is given by

$$
\gamma_{m}=\frac{p_{m} g_{m}}{P} \Leftrightarrow p_{m} g_{m}=P \gamma_{m} .
$$

Hence, (2) and (3) yield

$$
P=P \sum_{m=1}^{M} \gamma_{m}+N \Longleftrightarrow \frac{P}{N}=\frac{1}{1-\sum_{m=1}^{M} \gamma_{m}},
$$

which provides the fractional load $L$ in (1), see also [15]. The dedicated physical data channel (DPDCH) is transmitted at a fixed power offset $\beta$ to the DPCCH power. Therefore, both these channels contribute to the fractional load:

$$
L=\sum_{m=1}^{M} \gamma_{m}(1+\beta)
$$

The interference cancellation capacity gain can be described by comparing the average fractional load for the same number of UEs, $M$, using either RAKE or PIC. Utilizing the average load for the two different receiver schemes,

$$
L^{\mathrm{RAKE}}=\frac{M}{M_{P}^{\mathrm{RAKE}}}, \quad L^{\mathrm{PIC}}=\frac{M}{M_{P}^{\mathrm{PIC}}},
$$

the average pole capacity gain is related via (6) by the average load for equal number of UE's as

$$
M_{P}^{\mathrm{PIC}}=\frac{L^{\mathrm{RAKE}}}{L^{\mathrm{PIC}}} M_{P}^{\mathrm{RAKE}} .
$$

Figure 4 illustrates the relation between the number of users and noise raise on average for both conventional RAKE and PIC. Furthermore, it is emphasized how the performance gain can both be seen as a capacity improvement given a fixed noise rise, or a coverage improvement due to a lower noise rise with PIC given a fixed number of users. 


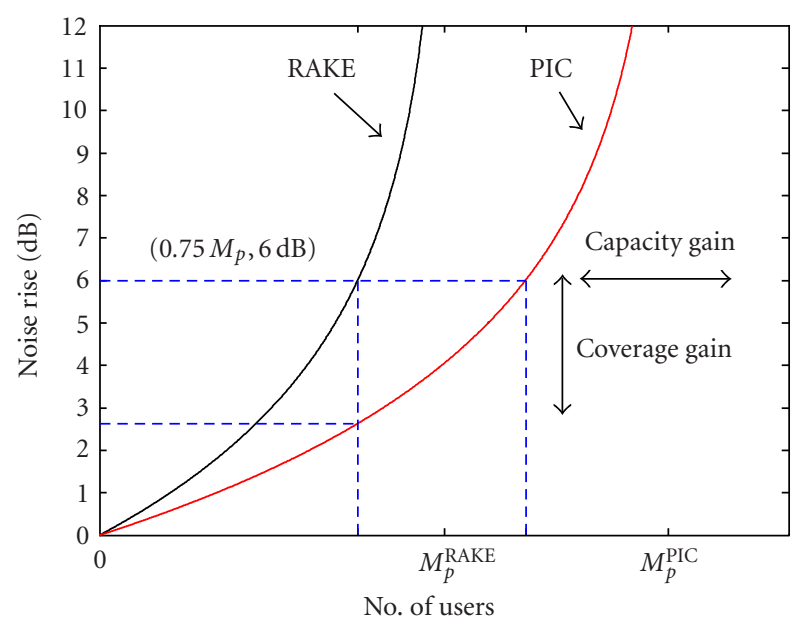

FIGURE 4: Illustration of the relation between noise rise and number of users for conventional RAKE and PIC.

\section{PARALLEL IC SIMULATION MODELING}

A dynamic system simulator is used to evaluate system capacity for a defined outage probability level. An outage occurs when a users' average service quality requirement is not supported. The outage probability is therefore estimated as the fraction of unsatisfied users relative to the total number of users in the entire simulation. The system simulator models the propagation environment, mobility, and traffic services. Radio resource control algorithms such as power control, soft handover, cell selection, and so forth are also included [16]. The PIC functionality is modeled as an intracell interference adjustment reflecting the effective received CIR as

$$
\gamma_{m, b}=\frac{p_{m, b}}{\sum_{i \neq m} \mathrm{RIF}_{i, b} \cdot p_{i, b}+\sum_{i, j \neq b} p_{i, j}+N},
$$

where $\gamma_{m, b}, p_{m, b}$, and $N$ are the CIR for the $m$ th user in the $b$ th cell, the received power from the $m$ th user in the $b$ th cell, and the background noise power (AWGN), respectively. The parameter residual interference factor $\left(\mathrm{RIF}_{m, b}\right)$ denotes the ratio between the equivalent intracell interference for the $m$ th user in the $b$ th cell after and before PIC execution and reflects the PIC performance. $\mathrm{RIF}_{m, b}$ is defined according to

$$
\operatorname{RIF}_{m, b}=\frac{\sum_{l=1}^{L F}\left|h_{m, b, l} \cdot d_{m, b}-\hat{h}_{m, b, l} \cdot \hat{d}_{m, b}\right|^{2}}{\sum_{l=1}^{L F}\left|h_{m, b, l} \cdot d_{m, b}\right|^{2}},
$$

where $h_{m, b, l}, d_{m, b}, \hat{h}_{m, b, l}, \hat{d}_{m, b}$, and $L F$ are the channel coefficient of the $l$ th path, the data symbol, the estimated channel coefficient, the estimated data symbol, and the number of channel delay paths, respectively. In the sequel, these quantities will be discussed generically, and the individual-user indices $m$ and $b$ are omitted for clarity.

The typical system simulation workflow is to first run detailed link simulations to determine relations between CIR and block error rate (BLER) statistics, and then use these models in system simulations to obtain the system capacity.

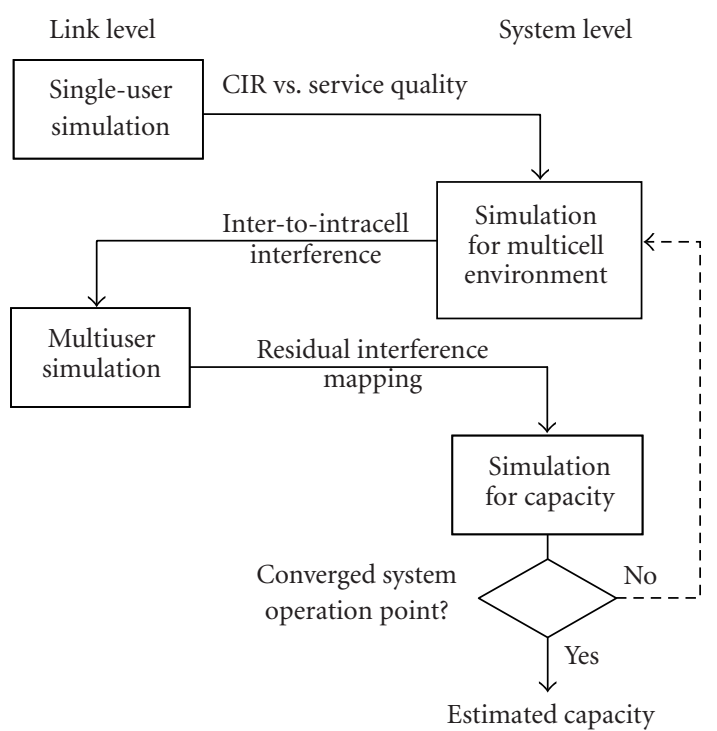

FIgUre 5: Workflow for PIC system evaluation.

The division between link- and system-level simulations is the selected approach to overcome the overwhelming complexity to simulate all the radio communication link details within a multiuser and multicell dynamic system environment, especially under realistic conditions with realistic algorithms. However, in this case, link simulations to obtain CIR and RIF mappings depend on the intercell interference situation, which is obtained from system simulations. The system simulations in turn depend on RIF to CIR mappings. Therefore, an iterative workflow as illustrated by Figure 5 is adopted, where iterated link and system simulations are performed to converge to the correct operation point of both the receiver performance and the impact through effective interference between connections. This will secure the PIC system interaction.

\subsection{Link-level simulation modeling}

The detailed link-level simulator can evaluate PIC performance under realistic conditions. Multiple users are simulated in a single cell, and intercell interference is modeled with an intercell-to-intracell interference ratio $F$. The main purpose is to provide a service quality and RIF mapping to the CIR. RIF is a multidimensional function of channel environment, $F$ (intercell-to-intracell interference ratio) value, target quality, number of active users, and PIC parameters such as weighting factors, DPCCH/DPDCH power ratio, and so forth. The link-level simulator calculates the RIF as in (9).

Realistic propagation channel conditions have been simulated using the COST 259 channel model [10], using the settings corresponding to a typical urban environment. The COST 259 channel model considers location-dependent channel variations such that a user moving continuously within a cell will experience different channel conditionspath loss, shadow fading, time dispersion, fast fading statistics, and so forth-in different parts of the cell. Each of these channel characteristics is described by physical parameters 
TABLE 1: Key characteristics of the COST 259 channel model.

\begin{tabular}{lcc}
\hline Characteristic & Model approach & Typical behavior \\
\hline Path loss & $\begin{array}{c}\text { COST 231 Walfisch-Ikegami model for non-line-of-sight } \\
\text { Distance-dependent probability for line-of-sight }\end{array}$ & $\begin{array}{c}\sim 40 * \log (d) \text { for non-line-of-sight } \\
\sim 20 * \log (d) \text { for line-of-sight }\end{array}$ \\
\hline $\begin{array}{l}\text { Clustering of multipath } \\
\text { components }\end{array}$ & $\begin{array}{l}\text { Multiple clusters caused by reflections } \\
\text { from distant buildings are modeled }\end{array}$ & $\begin{array}{c}\text { 13\% probability of } \\
\text { more than one cluster }\end{array}$ \\
\hline Shadow fading & Lognormal, exponential autocorrelation function & $6 \mathrm{~dB}$ \\
\hline Fast fading & $\begin{array}{l}\text { Rayleigh fading of channel taps, } \\
\text { first tap may be Rice fading }\end{array}$ & $\begin{array}{l}\text { Classical Doppler spectrum; higher } \\
\text { Rice factors in line-of-sight }\end{array}$ \\
\hline Time dispersion & $\begin{array}{l}\text { Exponentially decaying power-delay profile; delay } \\
\text { spread is modeled by a lognormal distribution }\end{array}$ & $0.2-1.0 \mu$ s RS delay spread \\
\hline Diversity & $\begin{array}{l}\text { Angular spread and polarization cross-coupling } \\
\text { give average power ratio and correlation }\end{array}$ & $\begin{array}{l}\text { Low cross-correlation, equal power } \\
\text { on diversity branches }\end{array}$ \\
\hline
\end{tabular}

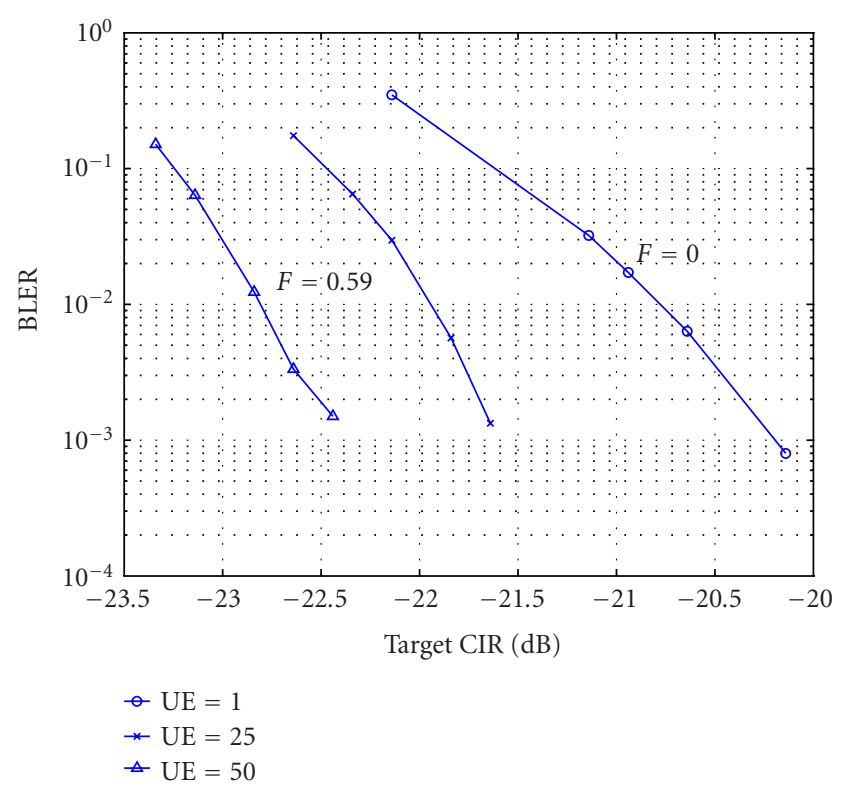

FIGURE 6: Link-level simulations of PIC performance. Multiple users are simulated in a single cell with intercell interference modeled as AWGN with the power $F$ times the intracell interference power. Note that UE $=1$ corresponds to conventional RAKE performance in a single cell.

that are modeled using statistical distributions. Correlations between links to different base stations have been added to the model for the system simulations. Further details on the characteristics of the model can be found in Table 1 .

Link-level simulations are conducted following the 3GPP specification [11] regarding physical parameters and protocols. The UL closed-loop power control model is CIR-based with a $1 \mathrm{~dB}$ step size, but including 1-slot feedback delay without feedback errors. The radio base station (RBS) receiver is configured with a 2-branch antenna diversity.

The PIC simulator model has also been carefully designed to be equivalent to the prototype implementation (i.e., functionality like path searcher, channel estimation, and so forth are modeled realistically). The intercell interference is emulated by additive white Gaussian noise with a power level according to the $F$ value. For link-level performance examples see Figure 6 and [17].

The presented link-level performance results in Figure 6 are simulated in a single-cell environment with an $F$-factor to model intercell interference. It represents examples of simulation results from both single- and multiple-user link-level simulations, which were pointed out in the simulation workflow in Figure 5. In Figure 6, exemplified for an AMR (adaptive multirate) speech user scenario, the BLER performance is shown as a function of the power control target $C I R$, that is, the outer-loop power control target for the fast innerloop PC handling the channel variations. Observe that the target CIR requirement for PIC decreases when the intracell interference to background noise ratio increases, that is, in a multiple-user environment. This can be seen in Figure 6 when the number of users increases from 1 to 50 with an equivalent increasing $F$ value of 0 and 0.59 , respectively. Note that the single-user case with PIC is equivalent to using a conventional RAKE receiver. The output from each batch of link-level simulations is a RIF to CIR mapping, at the operation points of interest, as exemplified in Figure $7 \mathrm{a}$.

\subsection{System-level simulation modeling}

System-level simulations are performed within a cellular structure configured with 3 cell sites deployment with cell radius of $1 \mathrm{~km}$. To avoid system border effects, a method of 7 -site cluster wrap around technique is utilized. The simulations are using a single WCDMA frequency carrier, and the soft handover functionality is limited to an active set equal to 3 (i.e., connections have maximum three legs). The UE transmit power dynamics is in the region -44 to $24 \mathrm{dBm}$. A fixed number of users are connected per simulation setup, and no load control such as admission/congestion control is activated.

The RIF mapping models PIC performance in the system simulations to determine the equivalent CIR after interference cancellation. Traditional CIR to block error probability (BLEP) models (see, e.g., [18]) are then used to determine 


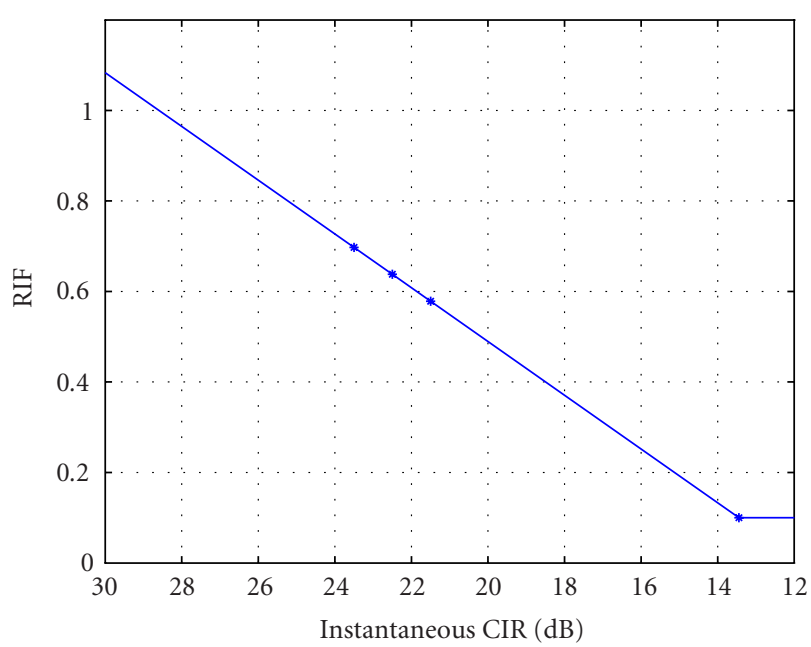

(a)

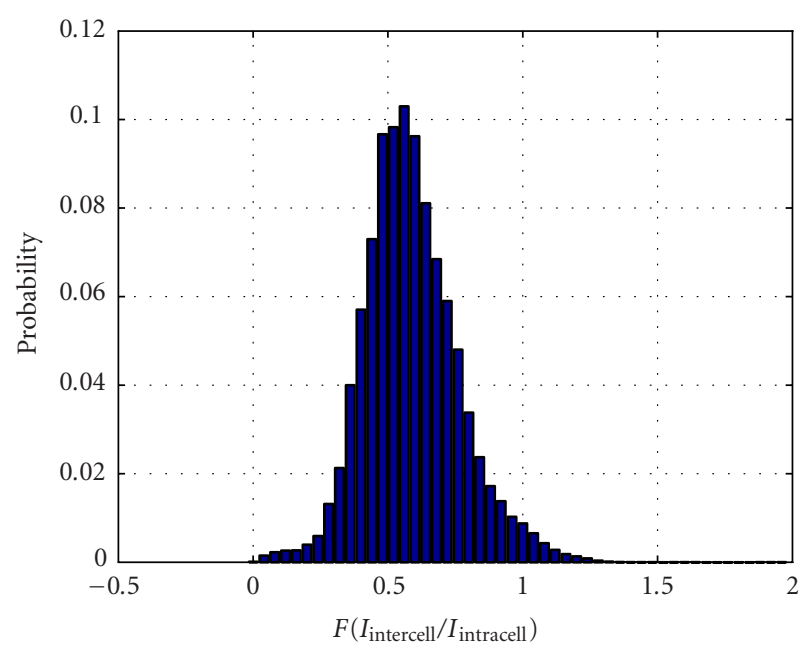

(b)

FIgURE 7: (a) RIF mapping and (b) F distribution over all cells.

transport block errors. (Note that BLEP is an error probability, while BLER is the error rate of the actual realization.) Outer-loop power control acts on the block error sequences to determine an appropriate target CIR for inner-loop power control to meet the BLER requirements [11, TS 25.214]. For example, the requirement of the considered AMR speech service is BLER $1 \%$. The main output from a batch of system simulations is an $F$ value distribution as exemplified in Figure $7 \mathrm{~b}$. The simulation workflow iterations in Figure 5 are halted when the RIF mapping and the $F$ distribution have converged, and the system capacity can be evaluated.

\section{SYSTEM PERFORMANCE EXAMPLE}

The uplink system performance is studied from the perspective of the capacity that one WCDMA carrier can support for each cell in the deployment. The system capacity is defined for a certain system quality level, that is, outage probability. In the performance examples shown in Figure 8, all users utilize AMR (12.2 kbps) speech service in a typical urban (COST 259) environment and slowly move around. The mobility model is a random walk with a mean velocity of $3 \mathrm{~km} / \mathrm{h}$. An AMR speech user is considered unsatisfied (is in outage) when the required average service quality of maximum $10^{-2}$ BLER is not satisfied. Reasons behind outages could typically be insufficient UE power to overcome the uplink interference. An outage probability (fraction of unsatisfied users) at $5 \%$ is considered acceptable, and the corresponding number of users in the system is therefore the system capacity. The system capacity in Figure 8 is normalized to the system capacity when the conventional RAKE receiver with realistic channel estimates is used in the system (Figures $8 \mathrm{a}$ and $8 \mathrm{~b}$ leftmost curve).

Using the above described quality measures, it can be concluded from Figure $8 \mathrm{~b}$ that PIC is supporting approximately a $40 \%$ uplink system capacity increase compared to utilizing the conventional RAKE receiver in this simulated realistic multiple-cell typical urban radio environment. Note that the PIC is carefully modeled in the simulations reflecting the limited-complexity implementation of realistic searcher, channel estimation, and so forth. In an equivalent singlecell environment setup, the realistic receiver implementation shows that PIC supports an improvement of the capacity in the order of $70 \%$ as indicated by Figure $8 \mathrm{a}$. The anticipated gain in capacity is expected to decrease for high-speed users [6].

As an upper bound regarded as reference of the technology limit, the outage performance of an ideal interference canceller (ideal IC) is also shown in Figure 8b (rightmost curve). An ideal IC receiver removes perfectly all intracell interference, equivalent to a RIF equal to zero in the CIR model in (8). For the ideal IC, the capacity increase is hence approximately $180 \%$ in the multiple-cell network environment with realistic channel estimates.

\section{SINGLE-CELL SYSTEM EVALUATION}

In a cooperative project between Ericsson, China Academy of Telecommunications Technology (CATT), and Datang Telecom Technology Co. Ltd., a PIC test system was developed and a field trial was performed at the northwest part of urban Beijing, China. The on-air field trial was performed during the period from December 2002 to May 2003. The trial system concept was based on a radio network controller (RNC) simulator connected to a modified commercial Ericsson RBS 3202 WCDMA radio base station providing PIC multiuser receiver functionality. The nonoptimized implementation of the PIC demodulator has about 5 times the complexity compared to a RAKE demodulator. However, note that this is comparable to a total receiver complexity, baseband part, increase of slightly less than 2 times. Pictures of the modified radio base station hardware employed for the tests can be found in Figure 9 and a map of the measurement area in Figure 10. 


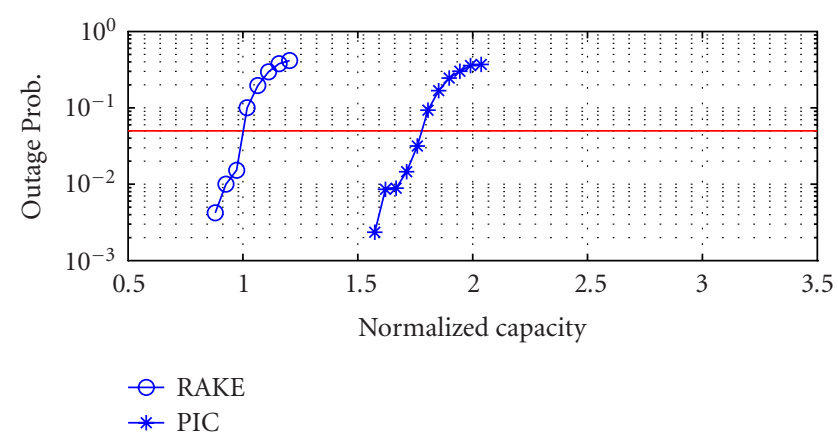

(a)

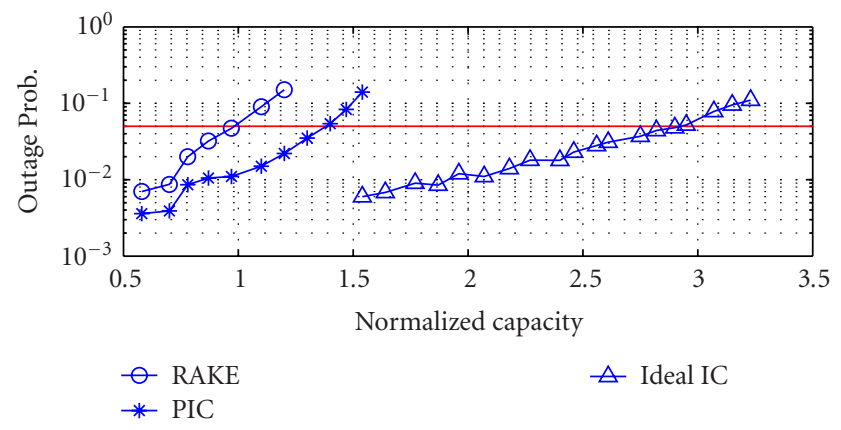

(b)

FIGURE 8: Simulated system performance relative to the performance with a conventional RAKE for (a) a single-cell scenario and (b) a multiple-cell scenario.

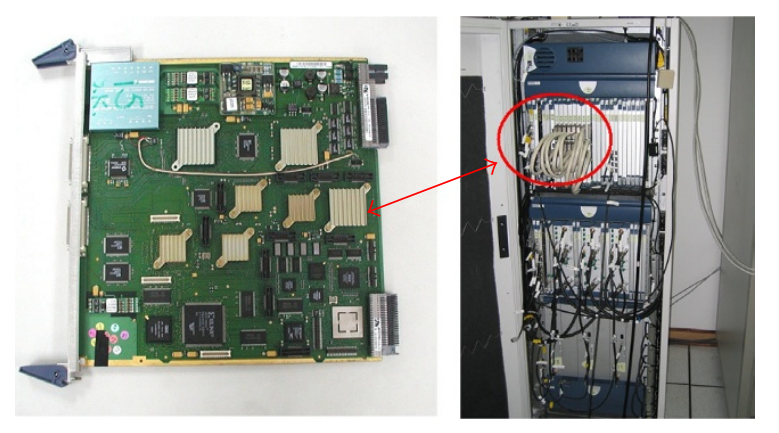

FIGURE 9: Modified Ericsson RBS 3202 hardware integrating multiuser PIC functionality.

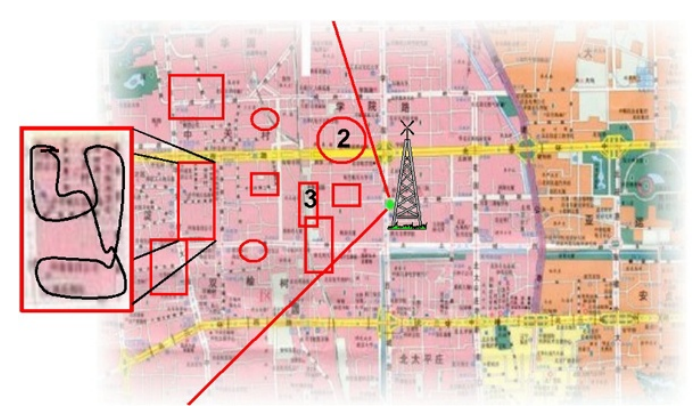

FIGURE 10: Defined measurement areas within RBS cell coverage.

The radio base stations were during all parts of the tests fully integrated into the WCDMA RAN infrastructure (i.e., connected to an RNC simulator using the 3GPP NBAP, Node B Application Protocol) supporting all layers 2 and 3 functionality with for example system information $(\mathrm{BCCH})$, paging and call setup handling, as well as the outer-loop power control. The field trial was performed in a single-cell system environment (Figure 10), which implies that no handover functionality was activated during the trial. The used prototype handset UEs were supporting 3GPP baseline, Release 99 December 2000, with AMR speech and 64 kbps UDI data [11].

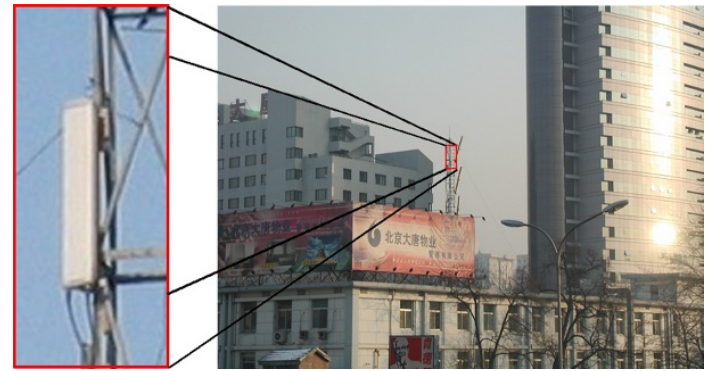

FIgURE 11: Antenna installation northwest Beijing, China.

The field trial measurement methodology is based upon the fact that UEs move/drive around in a for each UE confined measurement area; see Figure 10. The UEs will follow the normal traffic flow on a predefined route (i.e., mimic normal-user operation/behavior).

As exemplified in Figure 10, within the test system coverage, a set of confined measurement areas was defined with a size in the range $\sim 50-500 \mathrm{~m} \times 50-500 \mathrm{~m}$. In each confined area, a measurement route was defined where the important parameters are the radio environment, angular direction, and distance. The different confined areas and routes are in some cases overlapping, and in addition, multiple UEs can be allowed within the same area during a test.

The radio base station antenna ( $17 \mathrm{dBi}$ gain) was installed at the rooftop of a three-floor building (see Figure 11) in northwest Beijing at approximately $25 \mathrm{~m}$ height. The $65-$ degree antenna boresight was west-north-west with a selected sector radii of $\sim 3 \mathrm{~km}$ overlooking an urban area including open areas and houses of both equivalent height as the installation as well as very much higher buildings.

The radio environment in the test system coverage area is exemplified in Figure 12, including measurements from the confined areas number 2 and 3 indicated in Figure 10. Shown in Figure 12 are the impulse responses as a function of time when moving on the dedicated route in the two areas. In conjunction to the impulse responses, the measured 


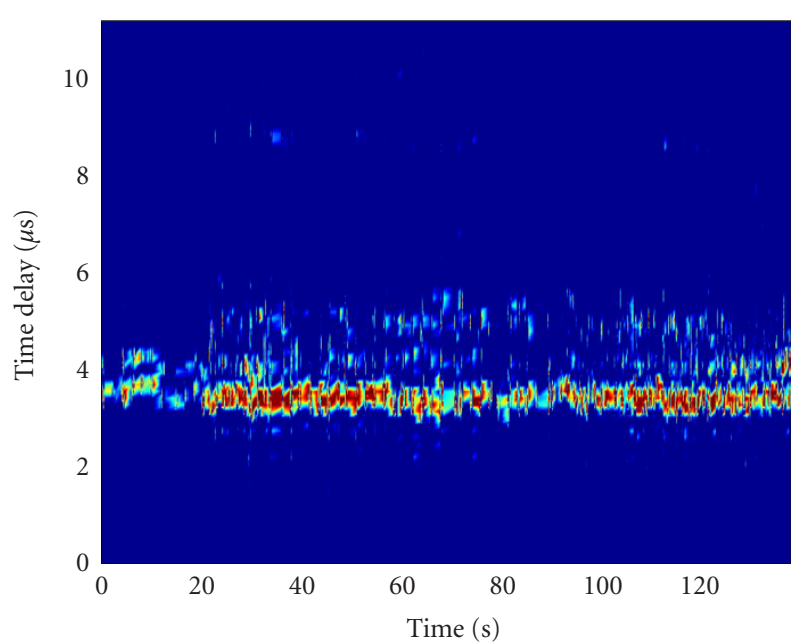

(a)

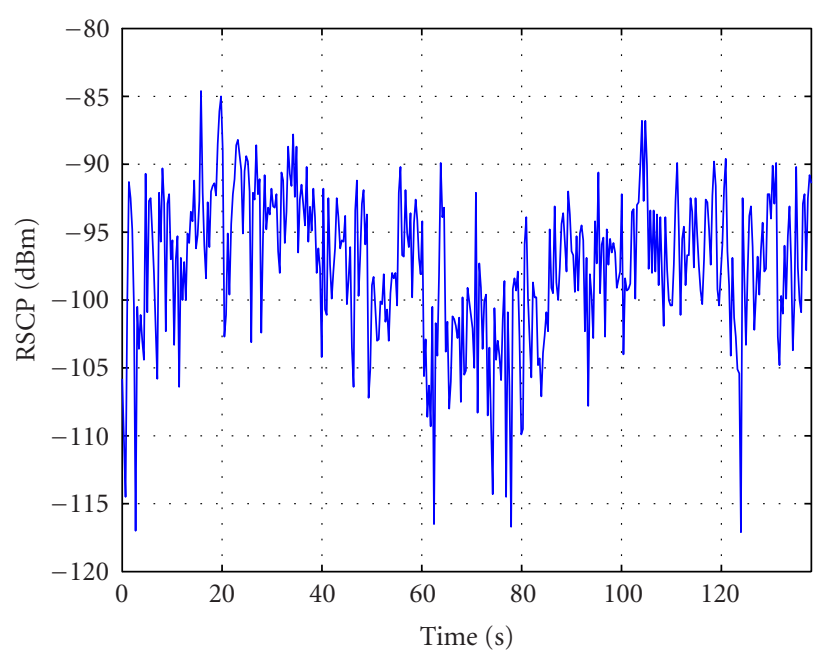

(c)

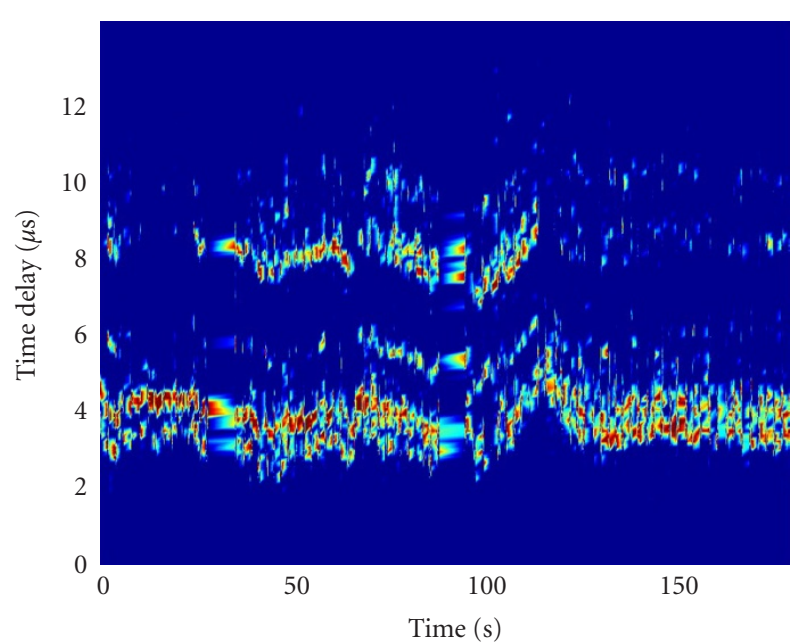

(b)

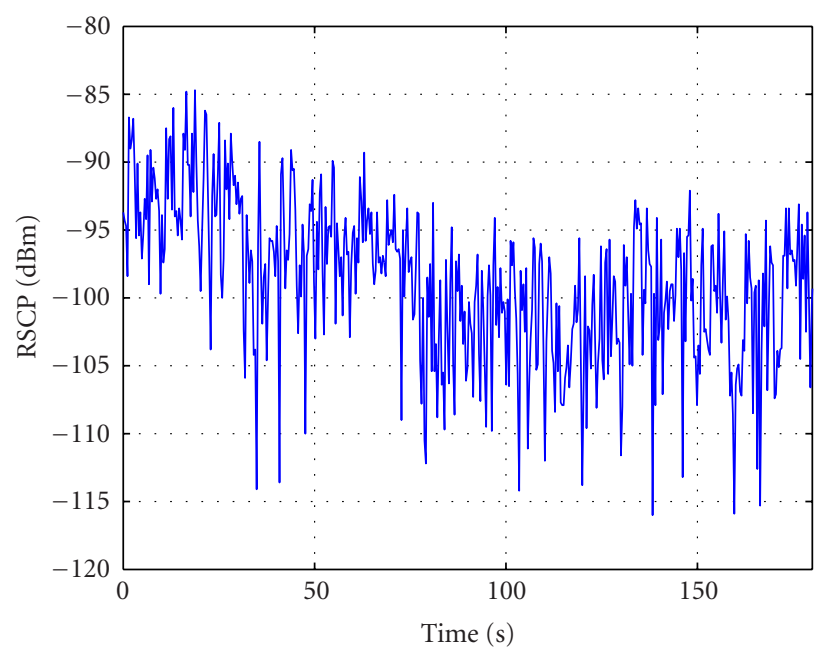

(d)

FIGURE 12: (a) and (b) Impulse responses and (c) and (d) received code power along the measurement routes in areas 2 and 3, respectively.

received code powers are presented when transmitting at a fixed UE power level $(24 \mathrm{dBm})$. The impulse response for area 2 (Figure $12 \mathrm{a}$ ) has mainly one dominating path while for area 3 , multiple paths are dominating. The measured impulse responses for the selected test system coverage area show that the trial environment can be classified as typical urban from a radio propagation modeling perspective.

The network integrated single-cell system performance test scenarios were conducted with different sets of multipleuser traffic and the results indicated that the implemented prototype PIC system provides performance (service quality, required UE power level, and so forth) and behavior improvements in accordance with expectations.

To exemplify the behavior improvement, results are shown in Figure 13 to Figure 16 from live on-the-air test scenarios with walking users in confined area 3. In Figure 13, a scenario with two communicating UEs was used, and in
Figure 14 to Figure 16, a scenario with four communicating UEs were used. In the execution for the different scenarios, the environment and service quality requirements resulted in different quality settings (Target CIR) from the layer 3 outerloop power control algorithm used per UE in the tests.

Each test scenario was set up and executed in an identical fashion twice. Between the executions, the only difference being that the PIC prototype test system was configured for utilizing either the conventional RAKE or the PIC receiver.

Figure 13 shows, for both test occasions, the outer-loop power control target CIR commands (the RNC layer 3 algorithm decisions) for the two active UEs when moving along the test route. Observe that a point-by-point comparison of the measurement values may not be valid even though the test setup and completion has been performed as similar as possible for the two test occasions. However, comparing the overall statistics, relative differences in the behavior can be 


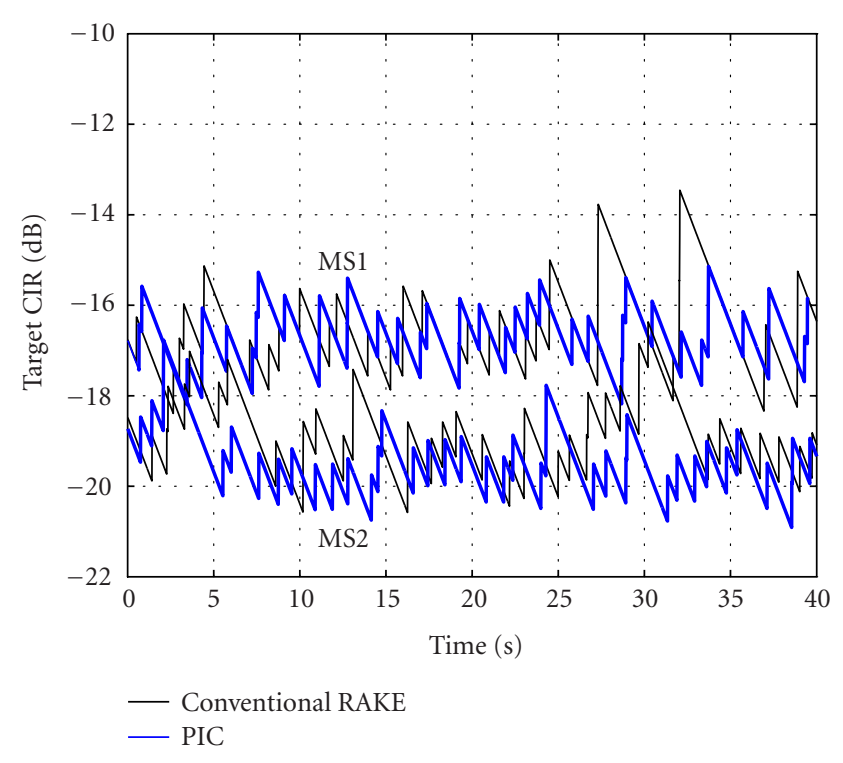

FIgURE 13: RNC (layer 3) target CIR commands when either conventional RAKE or PIC receiver is utilized for users 1 and 2 .

found. When examining and comparing the specific measured scenario in Figure 13, the measurement results imply that the PIC receiver improves the control process stability compared to a system configuration utilizing the conventional RAKE receiver. The statistics for the two cases show a reduction in mean and standard deviation of the SIR target. For UE 1, the mean values are reduced from $3.43 \mathrm{~dB}$ to $3.3 \mathrm{~dB}$ and the standard deviation from $0.938 \mathrm{~dB}$ to $0.601 \mathrm{~dB}$ when the PIC is active. For UE 2, the mean values are reduced from $1.08 \mathrm{~dB}$ to $0.499 \mathrm{~dB}$ and the standard deviation from $0.802 \mathrm{~dB}$ to $0.677 \mathrm{~dB}$ when the PIC is active. For this measured scenario, this implies an overall increased capacity (less interference generated) and an increased battery life of the UE terminal. Since the outer-loop power control is measuring and controlling the service quality over all 3 layers in the radio access network (RAN) and adjusts the targets, the decrease in standard deviation indicates a stabilizing effect on the system.

Figures 14 and 15 illustrates the outer-loop power control target CIR commands (the RNC layer 3 algorithm decisions) for the active UEs when moving along the test route during call setup with four UEs. The setup phase is selected to illustrate the improved power control stability due to PIC. Essentially, the target CIR variations are related to the uplink load, and therefore, the load reduction due to PIC also improves system stability. This is evident from Figure 16, which provides cumulative distribution functions of the uplink load for RAKE and PIC, respectively, for the case of four active UEs. Clearly, PIC implies a more stable system.

Corresponding system load as defined by (1) and computed in (5) for single-cell networks is used to compare the resulting load in the two tests (RAKE and PIC, respectively) when all UEs are connected. Then the estimated capacity

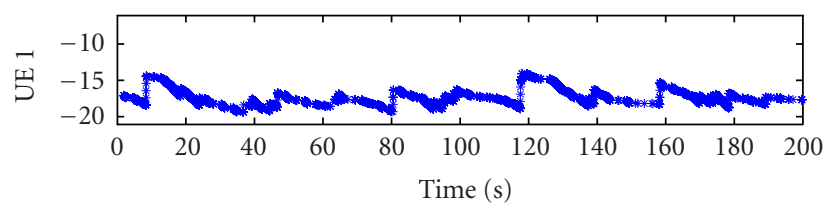

(a)

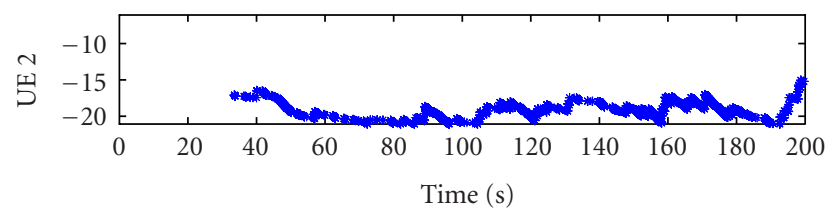

(b)

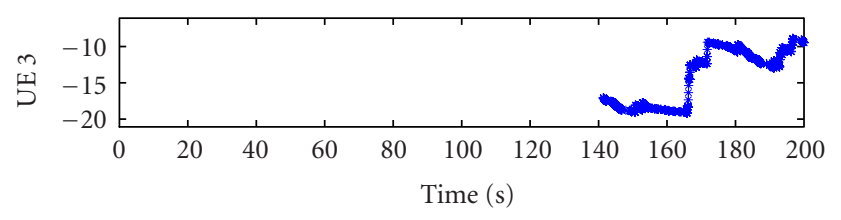

(c)

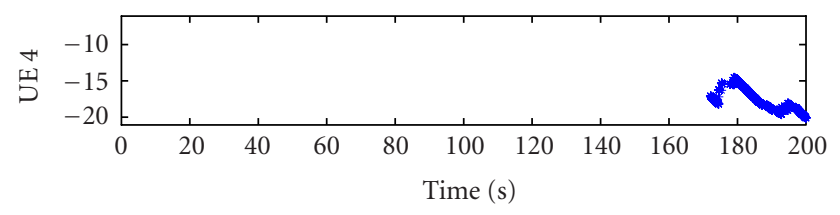

(d)

FIGURE 14: RNC (layer 3) target CIR commands with conventional RAKE.

gain can be evaluated as in (7):

$$
\text { Capacity gain : } \frac{\text { mean } L^{\mathrm{RAKE}}}{\text { mean } L^{\mathrm{PIC}}} \approx 1.65 \text {. }
$$

This is in accordance with observations from the realistic simulations in Section 5.

\section{CONCLUSION}

The main conclusion that can be drawn from the extensive work that has been carried out, involving link- and systemlevel simulations and field trials, is that there are major uplink performance gains achievable even so for interference cancellation base station architectures of rather limited complexity. This is primarily due to the known nature of the inherent intracell interference generated in WCDMA networks, which can be exploited by the IC technology to offer a large theoretical uplink gain.

The limited-complexity PIC receiver supports a $40 \%$ uplink network capacity increase for walking speech users in the simulated realistic typical urban radio environment. In the simulations, the PIC is carefully modeled to reflect realistic implementations of searcher, channel estimation, and 


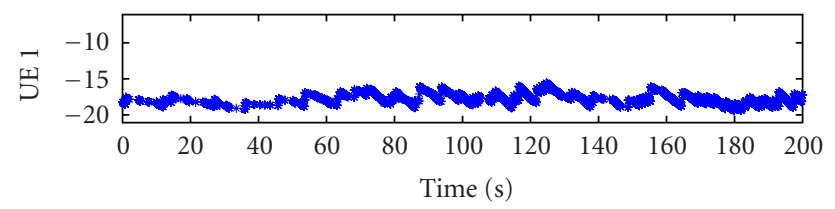

(a)

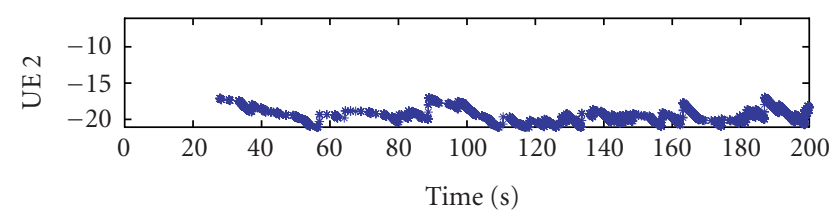

(b)

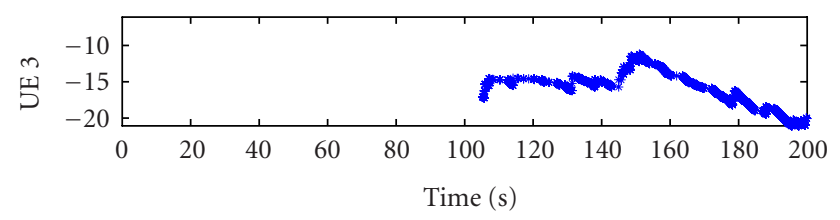

(c)

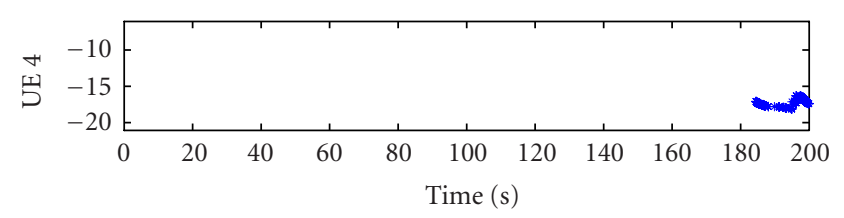

(d)

FIgure 15: RNC (layer 3) target CIR commands with PIC.

so forth and the on-the-air trial results indicate that the implemented prototype PIC system provides performance and behavior improvements in accordance with expectations.

Observations of the on-the-air trial measurement dynamics imply that the PIC receiver stabilizes the control process via a reduction in standard deviation of the RNC outerloop PC target CIR commands. The single-cell estimated capacity gain in the order of $70 \%$ might potentially be experienced in inhomogeneous deployments with isolated highdemanding hotspot cells. Moreover, the estimated capacity gain for single-cell systems is in accordance with results from realistic network simulations. This also indicates that the simulation models are relevant and representative.

\section{ACKNOWLEDGMENTS}

Needless to say, all the "background" work that has been carried out to design and build a prototype system, and to set up and perform the field measurements, has involved quite a lot of people. The authors would in particular like to thank the persons of all collaboration partners in Beijing, Ericsson Radio Network R\&D Center Beijing, China Academy of Telecommunications Technology (CATT), and Datang Telecom Technology Co. Ltd., for their excellent effort and the

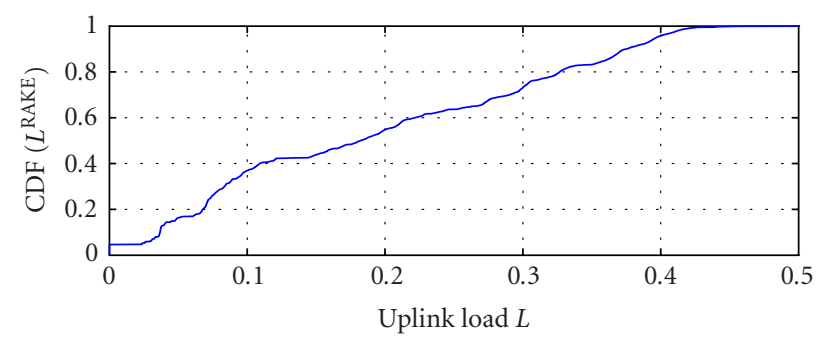

(a)

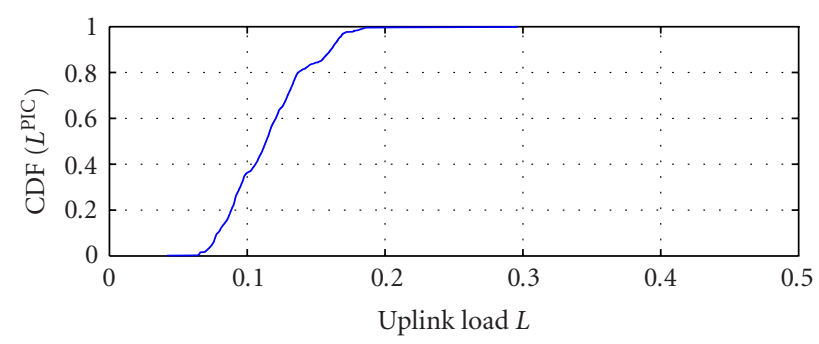

(b)

FIGURE 16: CDF comparison of the uplink load for (a) RAKE and (b) PIC.

magnificent fruitful cooperation. Furthermore, we are also very grateful to all the other persons that has been involved for critical support from various organizations and places: Ericsson Research in Tokyo, Linköping, Jorvas, Budapest, and Kista; Ericsson R\&D in Beijing, Mölndal, Kista, and Enschede; TietoEnator in Göteborg, Karlstad, and Umeå.

\section{REFERENCES}

[1] S. Verdú, "Minimum probability of error for asynchronous Gaussian multiple-access channels," IEEE Trans. Inform. Theory, vol. 32, no. 1, pp. 85-96, 1986.

[2] R. Lupas and S. Verdú, "Linear multiuser detectors for synchronous code-division multiple-access channels," IEEE Trans. Inform. Theory, vol. 35, no. 1, pp. 123-136, 1989.

[3] P. Patel and J. Holtzman, "Analysis of a DS/CDMA successive interference cancellation scheme using correlations," in Proc. IEEE Global Telecommunications Conference (GLOBECOM '93), pp. 76-80, Houston, Tex, USA, December 1993.

[4] M. K. Varanasi and B. Aazhang, "Multistage detection in asynchronous code-division multiple-access communications," IEEE Trans. Commun., vol. 38, no. 4, pp. 509-519, 1990.

[5] T. Shima, R. Esmailzadeh, J. Karlsson, and T. Yamauchi, "User capacity of a single cell WCDMA system with different IQ power ratios with and without interference cancellation," in Proc. IEEE CDMA International Conference and Exhibition (CIC '99), pp. 36-39, Seoul, Korea, September 1999.

[6] M. Ariyoshi, T. Shima, J. Han, J. Karlsson, and K. Urabe, "On the effect of forward-backward filtering channel estimation in W-CDMA multi-stage parallel interference cancellation receiver," IEICE Transactions on Communications, vol. E85-B, no. 10, pp. 1898-1905, 2002.

[7] M. Sawahashi, K. Higuchi, H. Andoh, and F. Adachi, "Experiments on pilot symbol-assisted coherent multistage interference canceller for DS-CDMA mobile radio," IEEE J. Select. Areas Commun., vol. 20, no. 2, pp. 433-449, 2002. 
[8] T. Suzuki, Y. Takeuchi, F. Watanabe, and S. Niida, "Implementation and field test result of a base station canceling CDMA interference for the IMT-2000 air interface," in IEEE 50th Vehicular Technology Conference (VTC '99), vol. 2, pp. 10031007, Amsterdam, The Netherlands, September 1999.

[9] T. Futami, H. Seki, and Y. Tanaka, "Performance evaluation of DS-CDMA multistage interference canceller in multi-cell environments," in Proc. IEEE Global Telecommunications Conference (GLOBECOM '98), vol. 1, pp. 247-252, Sydney, NSW, Australia, November 1998.

[10] L. M. Correia, Ed., Wireless Flexible Personalized Communications-COST 259: European Co-operation in Mobile Radio Research, John Wiley \& Sons, Chichester, England, UK, 2001.

[11] 3GPP Technical Specification Group Radio Access Network, Release 99, December 2000, available from http://www.3gpp.org/ftp/Specs/2000-12/R1999/, specifically 23-series, 24-series and 25-series.

[12] D. Divsalar, M. K. Simon, and D. Raphaeli, "Improved parallel interference cancellation for CDMA," IEEE Trans. Commun., vol. 46, no. 2, pp. 258-268, 1998.

[13] N. S. Correal, R. M. Buehrer, and B. D. Woerner, "A DSPbased DS-CDMA multiuser receiver employing partial parallel interference cancellation," IEEE J. Select. Areas Commun., vol. 17 , no. 4, pp. 613-630, 1999.

[14] H. Holma and J. Laakso, "Uplink admission control and soft capacity with MUD in CDMA," in IEEE 50th Vehicular Technology Conference (VTC'99), vol. 1, pp. 431-435, Amsterdam, The Netherlands, September 1999.

[15] E. Geijer Lundin, F. Gunnarsson, and F. Gustafsson, "Uplink load estimation in WCDMA," in Proc. IEEE Wireless Communications and Networking Conference (WCNC' 03), vol. 3, pp. 1669-1674, New Orleans, La, USA, March 2003.

[16] M. Karlsson, M. Almgren, S. Bruhn, K. Larsson, and M. Sundelin, "Joint capacity and quality evaluation for AMR telephony speech in WCDMA systems," in IEEE 56th Vehicular Technology Conference (VTC '02), vol. 4, pp. 2046-2050, Vancouver, Canada, September 2002.

[17] H. Murai, B. Hagerman, M. Tadenuma, et al., "System performance for WCDMA Up-Link interference cancellation - simulated results and field measurements," in Proc. IEEE International Symposium on Wireless Personal Multimedia Communications, Yokosuka, Kanagawa, Japan, October 2003.

[18] H. Olofsson, "Improved quality estimation for use in simulation of wireless systems," in Proc. IEEE International Conference on Universal Personal Communications, San Diego, Calif, USA, October 1997.

Bo Hagerman received the M.S.E.E., Lic. Tech. E.E., and Ph.D. degrees in radio communication systems from the Royal Institute of Technology (KTH), Stockholm, Sweden, in 1987, 1993, and 1995, respectively. From 1987 to 1990, he was a member of the technical staff at the Ericsson Radio Systems Research and Development Department, where he worked in the area of signal processing with applica-

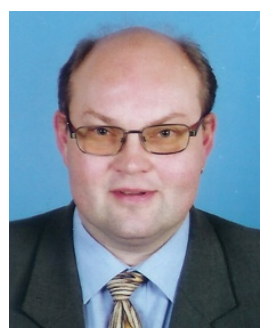
tions to GSM receivers. He joined the Department for Radio Access and Antenna Systems Research at Ericsson Research, Stockholm, in 1995, where he has been working in research on adaptive antennas in cellular systems. In 1999, he was appointed as the Senior Specialist in the area of advanced antenna systems.
His current research interest is in the areas of statistical signal and array processing for wireless communications and of radio resource management for cellular systems.

Fredrik Gunnarsson is a Senior Research Engineer at Ericsson Research, and a Research Associate at Linköping University, Sweden. He obtained his Ph.D. degree in electrical engineering from Linköping University in 2000. His research interests include radio resource management and signal processing for wireless communications.

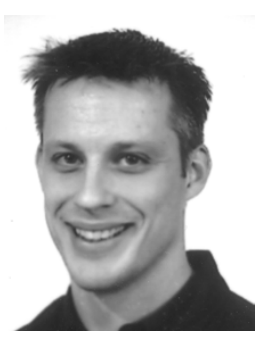

Hideshi Murai received the B.E. degree and the M.S. degree from the University of Electro-Communications, Tokyo, Japan, in 1983 and 1985, respectively. He also received the Ph.D. degree from Osaka University, Osaka, Japan, in 1988. During 1988-2000, he worked for Mitsubishi Electric Corporation in the area of digital satellite communications, spread-spectrum communications, and radio access technologies such as PDC and CDMA. Since 2000, he has been working for Ericsson Research in radio access technologies focused on $3 \mathrm{G}$ and beyond. During 2000-2001, he worked in Sweden as a Visiting Researcher from Nippon Ericsson. He is a Member of IEICE and IEEE.

Mioko Tadenuma was born in Kanagawa, Japan, on January 27, 1976. She received the B.E. and M.E. degrees in electrical engineering from Keio University, Japan, in 1998 and 2000. In 2000, she joined Ericsson Research in Japan. Her research interests include radio access technologies and interference cancellation.

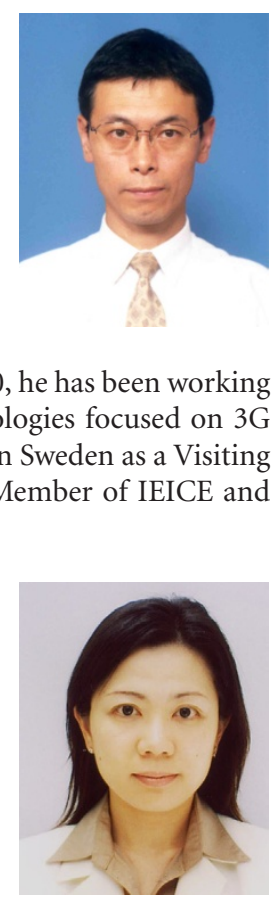

Jonas Karlsson was born in Örebro, Sweden, on June 30, 1969. He received the M.S. degree from Linköping University, Sweden, in 1993, the Licentiate degree from Royal Institute of Technology, Sweden, in 1998, and the Ph.D. degree from the University of Tokyo in 2003, all in electrical engineering. From 1993 to 1998, he worked for Ericsson Research in Sweden as a Research Engineer. In 1998, he joined Ericsson Research

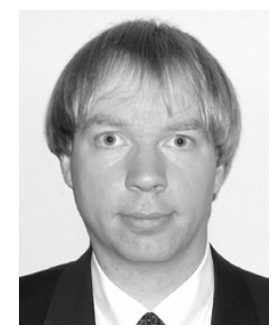
in Japan, where he has worked as a Research Manager since 2000. In 2004, he rejoined Ericsson Research in Sweden as a Senior Research Engineer. His research interests include radio access technologies, advanced antennas, and interference cancellation. 